\title{
Screening of Diabetic Patients for Frailty with the Frail Scale: A Comparison with the Fried's Phenotype Criteria in Saudi Arabia
}

\section{Sadiq Ahmed Al-Ali ${ }^{1}$, Qasem Mohammed AlJabr ${ }^{2,3,4,5}$, Zainab Tariq Alramadhan $^{6}$, Zainab Algharrash $^{6}$, Alhawraa Jassim Ahmad Alyousif ${ }^{6}$, Aroob Nassir Alessa ${ }^{6}$ and Hassan Ali AlButayan ${ }^{6}$}

\author{
${ }^{1}$ Family Medicine Senior Registrar, Saudi Board of Family Medicine, Postgraduate Center of Family \\ Medicine, Ministry of Health, Alahsa Province, Saudi Arabia \\ ${ }^{2}$ American Board Certified in Family Medicine, Texas Tech University, USA \\ ${ }^{3}$ American Board Certified in Geriatric Medicine, University of Miami/JMH, USA \\ ${ }^{4}$ Clinical Research Fellow, George Washington University, USA \\ ${ }^{5}$ Consultant of Family Medicine and Geriatrics, Staff in Postgraduate Center of Family and Community \\ Medicine, AlAhssa, Saudi Arabia \\ ${ }^{6}$ Medical Doctor MBBS, Primary Health Care Ministry of Health, AlAhssa, Saudi Arabia
}

*Corresponding author: Dr. Sadiq Ahmed Al-Ali, Family Medicine Senior Registrar, Saudi Board of Family Medicine, Postgraduate Center of Family Medicine, Ministry of Health, Alahsa Province, 2708 Albuhturyst - South Hofuf City, 820336443, Saudi Arabia, Tel: +966562534888

\section{Abstract}

Background and aims: Frailty is a serious health issue that is associated with the decline of muscle and nerve functions. Several conditions have been associated with frailty, such as dementia, cancer, and diabetes. The aim of the study is to evaluate the prevalence of frailty in Saudi diabetic patients and assess the association between diabetes and frailty. Also, to investigate if the FRAIL scale is an adequate tool to identify frail patients to pre-frail and healthy patients.

Methods: Seventy-eight participants were divided into three categories; group-I, the control group, included 31 healthy men 65 to 75 years, group-II 25 patients with DM2 aged $50-64$, and group-III included 22 patients with DM2 aged 65-80 years-old. Frailty index using Fried's criteria (CHS) and FRAIL scale was determined for all the participants. In ROC analysis, we evaluated diagnostic accuracy and AUC areas of the FRAIL scale compared with the CHS criteria.

Results: Frail patients were more in Group II and III (diabetics) $(44 \%, 55 \%)$ respectively than in Group-I (nondiabetics) $10 \%$. People aged 63 -years-old or more are more likely to have frailty syndrome.
\end{abstract}

Diabetic patients with FBG level $\geq 220 \mathrm{mg} / \mathrm{dL}, \mathrm{HbA} 1 \mathrm{c}$ level $\geq 9.5 \%$, are more likely to be frail patients (P-values: 0.029 , 0.002 ) respectively. Comparisons of the FRAIL scale to CHS components showed an independent diagnostic property.

Conclusion: Frailty is a very serious condition, and it's a leading cause of morbidity and mortality. FRAIL scale is a promising diagnostic tool, and more diagnostic studies are recommended.

\section{Introduction}

Frailty is considered as a geriatric condition [1,2]. It leads to a decline in muscle and nerve functions and loss of cardiopulmonary function [3]. It is the most serious health issue in the world and the major risk factor for morbidity and mortality in the elderly [4]. Frailty is an impairment in multiple physiologic systems, which leads to a limitation in physical function [1,5-11]. There are many other risk factors associated with frailty including socioeconomic factors such as poverty and low educational level; psychologic factors 
such as depression, and nutritional factors; and also some diseases such as cancer, dementia and endocrine disorder like diabetes mellitus [5,7,12-15]. Several studies showed that frailty is more in older diabetic patients, which results in increasing the mortality in frail than non-frail diabetic patients [16-19].

Frailty is independent of age, although the prevalence increases with it [20]. In United States, the prevalence of frailty is estimated to be between $4.0-59.1 \%$ and when tested, especially among diabetic patients, is estimated to be between $5-48 \%[21,22]$.

Globally, there are a limited number of studies that were conducted to assess the prevalence of frailty in elderly diabetic people. One of these studies was done among the Mexican American population in Texas, and it revealed that diabetes significantly contributes to increased frailty prevalence in older adults.

In 2001, more than 27 definitions were published to diagnose frailty $[23,24]$. The frailty phenotype by fried and colleagues the (Cardiovascular Health Study Index [CHS]) [2] and the Frailty Index (FI) by Mitnitski and colleagues [17] are the most two strategies used to diagnose frailty ( $69 \%$ and $12 \%$ of published studies, respectively) [24].

However, both strategies (CHS and $\mathrm{Fl}$ ) are challenging to use in clinical settings because they need trained staff to do it. An adequate way used to screen frailty is a simple method using simple instruments, while the complex ones are preserved for specialized geriatric services [25].

There is no study aimed at evaluating the association between diabetes and frailty in Saudi Arabia; despite the increased number of diabetic patients. Therefore, this study is constructed and established to estimate the frailty index in Saudi diabetic patients using CHS score and compared it with the pre-elderly diabetics and agematched healthy population. Also, to investigate the diagnostic accuracy of FRAIL SCALE with CHS score (as gold standard in this study).

\section{Methods}

The present study included 78 men who attended the Chronic Diseases Clinics, Al-Ahsa, from December 2018 to January 2019. The participants had the following inclusion criteria: Age $>50$ years for diabetic participants and $>65$ years for non-diabetic participants, stable medical condition over the past month, and regular clinical follow-up. Subjects were excluded if they had dementia, Parkinson's disease, severe depression, or could not complete this study's procedures. Participants were divided into three groups. Group I included 31 non-diabetic participants between the age of 65 and 75 years and identified as the control group; group II included 25 patients with type 2 DM aged $50-64$ years; and group III included 22 patients with type 2 DM 65-
80 years-old. Written consent has been obtained from participants after informing them of the benefit and purpose of this study.

An extended and detailed medical history was obtained from each participant of groups II and III, emphasizing the onset, duration, and treatment of diabetes. Lab results have been seen from patients' files for possible additional diagnoses such as myopathy diseases or low testosterone hormone levels.

\section{The FRAIL scale}

The FRAIL scale includes five simple questions $[26,27]$. The FRAIL scale is based on self-report without any objective measurement. The five questions require a yes or no answer, with 1 point given to any affirmative response. Four of them were based on the Fried index criteria components [2], and one (i.e., number of illnesses) was based on the Frailty Index (FI) by Mitnitski and colleagues [28]. The score ranges from 0 to 5 points, and based on the score individuals can be classified as non-frail ( 0 points), prefrail (1 to 2 points), or frail (> 3 points). The scale evaluates the presence of fatigue, muscle resistance, aerobic capacity, disease burden, and weight loss. Fatigue is evaluated by asking participants if they felt tired most of the time; muscle resistance is measured by participants' report on his or her capacity to climb a flight of stairs; aerobic reserve is evaluated by participants' report on his or her ability to independently walk a block; disease burden by the presence of 5 or more of a total of 11 diseases (i.e, diabetes mellitus, hypertension, cancer, chronic obstructive pulmonary disease, coronary artery disease or myocardial infarction, stroke, congestive heart failure, asthma, chronic renal failure, arthritis,); and unintentional weight loss by $5 \%$ or more within the past 6 months.

\section{The cardiovascular health study index or fried's frailty phenotype (CHS)}

The CHS frailty phenotype includes five objective components (i.e., reduced grip strength, unintentional weight loss, self-reported exhaustion, self-reported low physical activity, and reduced gait speed) assessed according to a predefined protocol [2]. According to the number of criteria they have, individuals are classified as follows: Frail $(>=3)$, prefrail (1-2), and non-frail (0). In this study, the $\mathrm{CHS}$ criteria measures were performed as follows and received 1 point if: Weight loss of $5 \%$ or more was reported in the last year; fatigue was reported according to two questions depending on the Center for Epidemiological Studies Depression Scale [29]; the patient was in lowest of physical activity adjusted for sex according to a weighted score of kilocalories expended per week; grip strength in the lowest quintile, adjusted for body mass index (BMI) and gender; and gait speed was in the lowest quintile, depending on needed time to walk a distance equals to 4.6 meters, adjusted 
for standing height and gender. Strength of grip was obtained by the mean measure of three attempts using a manual hydraulic hand grip device (Jamar model) with the elbow at a 90-degree angle, with a brief rest between measurements, and with verbal stimulation during the measurement.

Group III was compared with their age-matched controls in group I, and group II was compared with group III. Frailty index was correlated with the duration of diabetes in groups II and III, and also with the glycemic level.

Frailty index using CHS score was determined for all participants; patients were categorized as frail when they attain three or more of the following parameters, pre-frail if they fulfilled one or two parameters, and non-frail if they did not attain any of the following parameters:

(a) Weight loss: Unintentional weight loss of at least $4.5 \mathrm{~kg}$ over the last year.

(b) Weakness (i.e., low hand-grip strength): Tested by grip strength of the dominant hand (mean of three measurements) using a Jamar hand-held dynamometer. The patient is asked to hold the dynamometer in the dominant hand, with the arm at right angles, and the elbow should be placed by the patient's side. The base rests on the first metacarpal, and the handle rests around the rest of the fingers. Once the patient is ready with the proper seating and the dynamometer's right grip, the participant should squeeze the device with maximum effort for five seconds. Meanwhile, the participant is not allowed to move any other body part. The cut-off points adjusted for BMI were (1) $\leq 32 \mathrm{~kg}$ for $\mathrm{BMI}>28$; (2) $\leq 30 \mathrm{~kg}$ for BMI 24.1-28; (3) $\leq 29 \mathrm{~kg}$ for BMI $\leq 24$.

(c) Fatigue or poor endurance: Depression scale is used in this study to evaluate poor endurance. The following two statements are specifically used for the evaluation: (a) 'I felt that everything I did was an effort' and (b) 'I could not get going'. The participant is determined as positive once he fulfills one at the minimum for at least three days over the last week.

(d) Slowness: Slowness is assessed by asking the participant to stand up from the chair, walk six meters distance, turn around, go back to the chair, and sit down again. The time of this process normally should be approximately 16 seconds.

(e) Low physical activity level: The level of physical activity is dependent on the participant's self-report. Those who have reported not doing daily activities, e.g., gardening, walking, or any sport over the last week, are considered physically inactive.

Participants were evaluated later for frailty with the FRAIL scale, and the scores for the two scales (FRAIL scale and $\mathrm{CHS}$ score) were compared.
Table 1: Characteristics of the participants involved in the study.

\begin{tabular}{|c|l|l|}
\hline Variable & Frequency & (\%) \\
\hline Gender & & \\
\hline Male & 43 & 55.1 \\
\hline Female & 35 & 44.9 \\
\hline Presence of diabetes & & \\
\hline Yes & 46 & 59 \\
\hline No & 32 & 41 \\
\hline
\end{tabular}

\section{Statistical Analysis}

Data were collected and coded and then entered into an IBM compatible computer using SPSS version 21. Addition to the obtained frequency tables, means and SDs were used to summarize data of categorical variables. Statistical significance was evaluated by Chisquare tests, with $\mathrm{P}$ values corrected using the Monte Carlo method for better precision. Receiver operating characteristic (ROC) analyses were performed to assess the diagnostic accuracy of the FRAIL scale and calculating areas under the curve (AUCs), sensibilities, specificities, positive predictive values, and negative predictive values. The CHS criteria were used as the gold standard to identify frailty status in accuracy analyses. Four items of the FRAIL scale were compared with correspondent components of the CHS by Chi-square test. The level of statistical significance was set at .05 level in 2-tailed tests.

Verbal consent from the participants has been taken before starting the study. It was clear to all participants that confidentiality and de-identification of data are respected in the study. Lastly, ethical clearance from the IRB committee in King Fahad Hospital, Al Ahssa, has been taken.

\section{Results}

A total of 78 participants were included in the present study, 43 of them were males (55.1\%), and 46 were diabetics (59\%) (Table 1). According to their age and diabetes status, participants were divided into three groups (Table 2). The age range in group I was 65-75 years, with a mean of $70.4 \pm 5.7$ years; the age range in group II was 50-64 years, with a mean of 58.4 \pm 2.8 years; and the age range in group III was $65-80$ years, with a mean of $71.6 \pm 4.9$ years. Participants of both groups II \& III were diabetic patients, with a range duration of DM 5-11 years, 11-15 years, respectively. Participants in group I were non-diabetics.

Regarding the evaluation of frailty status using $\mathrm{CHS}$ score, in group I, twenty patients (65\%) were non-frail, eight $(25 \%)$ were prefrail, and three $(10 \%)$ were frail; in group II, eight patients (32\%) were non-frail, six (24\%) were prefrail, and eleven (44\%) were frail; and in group III, four patients (18\%) were non-frail, six $(27 \%)$ were prefrail, and twelve (55\%) were frail (Table 3). There was 
Table 2: Distribution of the studied groups according to age (years).

\begin{tabular}{|l|l|l|l|l|l|l|}
\hline Groups & N & Age range & Mean \pm SD & Duration of DM & FBG (mg/dL) & HbA1c (\%) \\
\hline Non-diabetic patients (Group I) & 31 & $65-75$ & $70.4 \pm 5.7$ & - & - & - \\
\hline Diabetic patients (Group II) & 25 & $50-64$ & $58.4 \pm 2.8$ & $5-11$ years & $213 \pm 44$ & $9.4 \pm 1.2$ \\
\hline Diabetic patients (Group III) & 22 & $65-80$ & $71.6 \pm 4.9$ & $11-15$ years & $217 \pm 44$ & $10.2 \pm 1.8$ \\
\hline
\end{tabular}

DM: Diabetes Mellitus; FBG: Fasting Blood Glucose; Hba1c: Glycated Hemoglobin

Table 3: Comparison between the studied groups regarding $\mathrm{CHS}$ score.

\begin{tabular}{|c|c|c|c|c|c|}
\hline \multicolumn{2}{|l|}{ Parameters } & Group I & Group II & \multicolumn{2}{|l|}{ Group III } \\
\hline \multicolumn{2}{|c|}{ Fried index score (CHS) } & $n(\%)$ & $n(\%)$ & \multicolumn{2}{|l|}{$n(\%)$} \\
\hline \multicolumn{2}{|l|}{ Nonfrail (0) } & $20(65)$ & $8(32)$ & \multicolumn{2}{|l|}{$4(18)$} \\
\hline \multicolumn{2}{|l|}{ Prefrail (1-2) } & $8(25)$ & $6(24)$ & \multicolumn{2}{|l|}{$6(27)$} \\
\hline \multicolumn{2}{|l|}{ Frail (3-5) } & $3(10)$ & $11(44)$ & \multicolumn{2}{|l|}{$12(55)$} \\
\hline \multicolumn{2}{|l|}{ Total } & $31(100)$ & $25(100)$ & \multicolumn{2}{|l|}{$22(100)$} \\
\hline \multicolumn{2}{|c|}{ Group I vs. Group II } & \multicolumn{2}{|c|}{ Group I vs. Group III } & \multicolumn{2}{|c|}{ Group I vs. Group III } \\
\hline Chi-square & P Value & Chi-square & P Value & Chi-square & P Value \\
\hline 9.466 & $0.009^{*}$ & 15.264 & $<0.001^{*}$ & 1.190 & 0.552 \\
\hline
\end{tabular}

${ }^{*}$ Correlation is significant at the 0.05 level.

Table 4: Correlation of CHS scores with the demographic data and degree of glycemic control.

\begin{tabular}{|c|c|c|c|c|}
\hline \multirow[t]{2}{*}{ Variables } & \multicolumn{2}{|c|}{ Fried index score (CHS) } & \multirow{2}{*}{$\begin{array}{l}\text { Pearson Chi- } \\
\text { Square }\end{array}$} & \multirow[t]{2}{*}{ P-value } \\
\hline & > 3 (Frail) n (\%) & $<3$ (Non-Frail) n (\%) & & \\
\hline \multicolumn{5}{|l|}{ Age } \\
\hline$<63$-year-old & $11(50)$ & $11(50)$ & 3.830 & $0.047^{*}$ \\
\hline$\geq 63$-year-old & $15(26.8)$ & $41(73.2)$ & & \\
\hline \multicolumn{5}{|l|}{ Gender } \\
\hline Males & $12(27.9)$ & $31(72.1)$ & 1.270 & 0.188 \\
\hline Females & $14(40)$ & $21(60)$ & & \\
\hline \multicolumn{5}{|l|}{ FBG } \\
\hline$<220 \mathrm{mg} / \mathrm{dL}$ & $9(34.6)$ & $17(65.4)$ & 4.776 & $0.029^{*}$ \\
\hline$\geq 220 \mathrm{mg} / \mathrm{dL}$ & $14(66.7)$ & $7(33.3)$ & & \\
\hline \multicolumn{5}{|l|}{ HbA1c } \\
\hline$<9.5$ & $5(23.8)$ & $16(76$ & & \\
\hline$\geq 9.5$ & $18(69.2)$ & $8(30 . \imath$, & & \\
\hline
\end{tabular}

FBG: Fasting Blood Glucose; Hba1c: Glycated Hemoglobin; ${ }^{*}$ Correlation significant at the 0.05 level.

a significant difference between groups I and II, groups I and III in favor of participants in groups II and III, more likely to be frail than patients in group I. (P-value: 0.009, $<0.001)$, respectively. Nevertheless, there was no significant difference between groups II and III (P-value: 0.552).

Concerning the effect of the variables on the $\mathrm{CHS}$ score, the analysis showed that frailty syndrome (CHS score $\geq 3$ ) is more likely to be present in participants who are 63-years-old or more (p-value: 0.047) (Table 4). Similarly, there was a significant correlation between the CHS score and degree of glycemic control, in favor of patients who have FBG level $220 \mathrm{mg} / \mathrm{dL}$ or more, HbA1c level $9.5 \%$ or more, are more likely to have score 3 or more (P-values: $0.029,0.002$ ) respectively. There was no significant difference in the score regarding gender.
Prevalence of frailty differed according to the FRAIL scale ( $n=33 ; 42.3 \%$ ) and CHS score ( $n=26 ; 33.3 \%$ ) (Table 5). Twenty-four participants were classified as being frail by both instruments. Participants classified as non-frail using the FRIED index were 32 in comparison to the FRAIL scale 22. The two instruments appeared to be independent in the diagnostic evaluation of the sample $(p=<0.001$ ) (Table 5 ).

In ROC analyses, FRAIL SCALE AUC, was $0.936(\mathrm{Cl}$ $=0.882-0.989$ ) (Figure 1). By the cut-off score of $>=3$ on the FRAIL scale, frailty was detected with a 0.923 sensitivity, and 0.827 specificity, positive likelihood ratio 5.3, and negative likelihood ratio 0.09 (Table 6).

\section{Discussion}

Aging is characterized by many syndromes and 
Table 5: Prevalence of frailty.

\begin{tabular}{|l|l|l|l|l|}
\hline \multirow{2}{*}{ FRAIL SCALE, $\mathbf{n}$ (\%) } & \multicolumn{3}{|c|}{ Fried index score (CHS) } \\
\cline { 2 - 5 } & Frail, $\mathrm{n}=26$ & Prefrail, $\mathrm{n}=20$ & Non-frail, $\mathrm{n}=32$ & P-value \\
\hline Frail & $24(92.3)$ & $5(25)$ & $4(12.5)$ & $<0.001$ \\
\hline Prefrail & $2(7.7)$ & $8(40)$ & $13(40.6)$ & \\
\hline Non-frail & $0(0)$ & $7(35)$ & $15(46.9)$ & \\
\hline
\end{tabular}

Table 6: Sensitivity, specificity, Predictive Values, and Likelihood ratios for identification of Frailty by using of FRAIL scale compared with CHS score as a Gold Standard .

\begin{tabular}{|l|l|l|l|}
\hline \multirow{2}{*}{ FRAIL SCALE } & \multicolumn{2}{c|}{ Fried index score (CHS) } \\
\cline { 2 - 4 } & $>3$ (Frail) & $3<$ (Non-frail) & Total \\
\hline Frail patient & 24 & 9 & 45 \\
\hline Non Frail patient & 2 & 43 & 78 \\
\hline Total & 26 & 52 & $\%$ \\
\hline & & $\%$ & \\
\hline Sensitivity & & 92.3 & \\
\hline Specificity & & 82.7 & \\
\hline Positive predictive value & 72.7 & \\
\hline Negative predictive value & 95.6 & \\
\hline Positive likelihood ratio & 5.3 \\
\hline Negative likelihood ratio & 0.09 \\
\hline
\end{tabular}

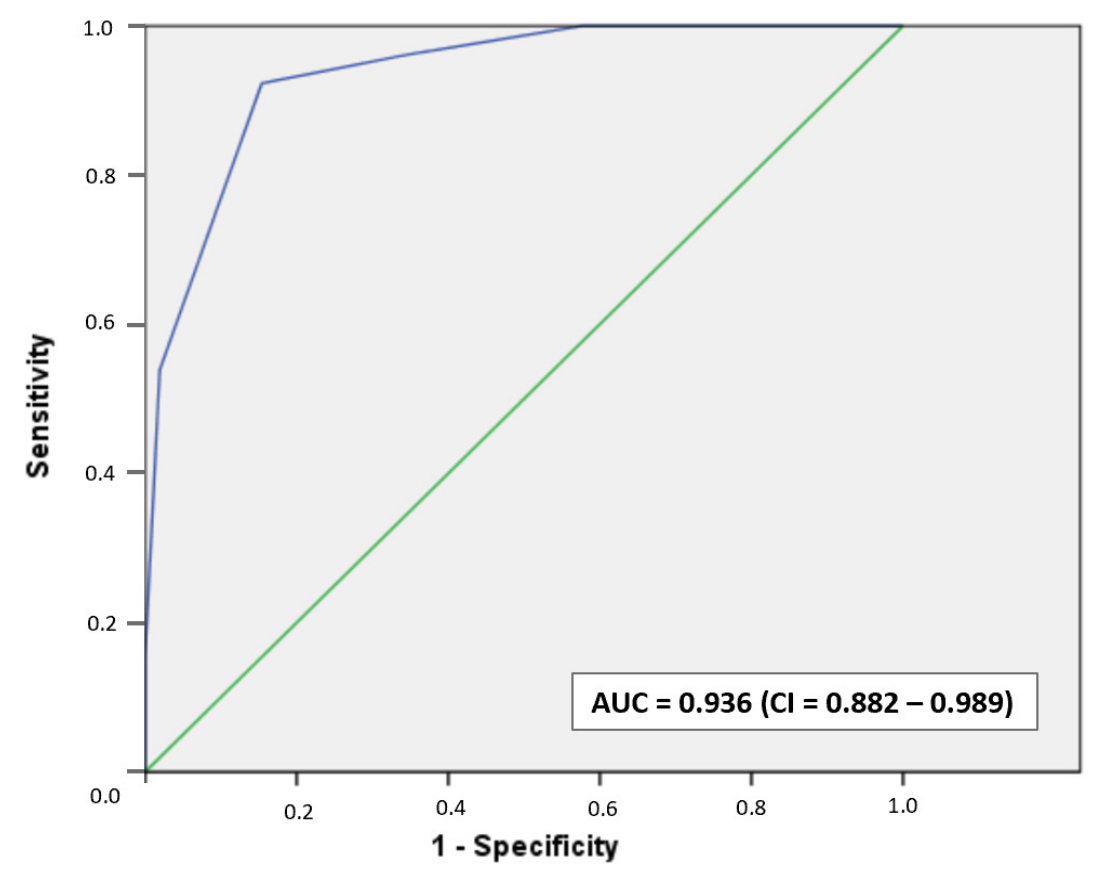

Figure 1: AUC for the detection of frailty by the FRAIL SCALE (Note: CHS score as the gold standard criteria for frailty).

frailty is one of the most serious aging problems [1,2], it is associated with poor prognosis including falls, high mortality and morbidities [1-3].

Diabetes mellitus type 2 plays major roles in accelerating the aging process, and diabetic patients are at higher risk of this syndrome [13].

The main aim if this study is to assess the correlation between frailty and diabetes in adult and older adults' patients in Alahsa - Saudi Arabia.
Seventy-eight patients enrolled in this study and divided into 3 groups. Group I included 31 healthy men between the ages of 65 and 75 years as a control group; group II included 25 patients with type 2 DM aged 50-64 years and group III included 22 patients with type $2 \mathrm{DM}$ who were $65-80$ years-old.

According to our data, the frailty score is higher among diabetic patients in groups 2 and 3 compared to those of non-diabetic patients in group 1 . However, 
we could not identify a significant correlation in terms if diabetic patients could develop frailty at an earlier age than non-diabetic patients.

On the other hand, a significant positive correlation was found between poor diabetic control and the score of the frailty. The study by Park, et al. screened 3075 older diabetic patients with muscle grip strength assessment and found muscle strength was lower in diabetic men compared to non-diabetic [30]. Many other studies suggest the correlation between diabetes mellitus type 2 and the onset of frailty and also recommends the screening of frailty in diabetic patients above age of $55[18,31,32]$. Some articles examine the use of frail scale as timed and cost-effective methods to assess for frailty but not enough data for accuracy $[33,34]$. In our article, we compared the frail scale to CHS score; however, the frail scale was reaching the sensitivity of $92.3 \%$ and specificity of $82.7 \%$. The positive likelihood ratio is 5.3 , while the negative likelihood ratio is 0.09. Our study supports screening diabetic patients for frailty; therefore, frailly screening could decrease frailty complications like falls and deaths. The frail scale could be used as a simple screening tool for frailty in Saudi patients.

Further studies with larger samples size needed to assess the prevalence of frailty in Saudi Arabia, assessing any possible correlation between diabetes and early onset of the frailty in addition to more diagnostic validation studies for the frail scale.

\section{Conclusion}

Frailty is a serious condition associated with very poor outcome; diabetes and frailty are related, especially among patients with poor glycemic control or medical comorbidities.

Frailty screening among diabetic patients is very important, and it could have a significant role in improving outcomes and avoiding life-threatening complications. Frail scale is a promising tool for screening but still lacks adequate diagnostic studies.

\section{Highlights}

- Frailty is a serious health issue that requires a high level of care.

- Diabetic patients have a higher risk of developing this syndrome.

- An accurate assessment tool is needed to detect patients early.

- Several outcomes related to frailty can be managed if the condition is detected early.

\section{References}

1. Morley JE, Vellas B, van Kan GA, Anker SD, Bauer JM, et al. (2014) Frailty consensus: A call to action. J Am Med Dir Assoc 14: 392-397.
2. Fried LP, Tangen CM, Walston J, Newman AB, Hirsch C, et al. (2001) Frailty in older adults: Evidence for a Phenotype. J Gerontol A Biol Sci Med Sci 56: M146-156.

3. Yanase T, Yanagita I, Muta K, Nawata H (2018) Frailty in elderly diabetes patients. Endocr J 65: 1-11.

4. Vaupel JW (2010) Biodemography of human ageing. Nature 464: 536-542.

5. Clegg A, Young J, lliffe S, Rikkert MO, Rockwood K (2014) Frailty in older people. Lancet 381: 752-762.

6. Gielen E, Verschueren S, O'Neill TW, Pye SR, O'Connell MDL, et al. (2012) Musculoskeletal frailty: A geriatric syndrome at the core of fracture occurrence in older age. Calcif Tissue Int 91: 161-177

7. Lang P-O, Michel J-P, Zekry D (2009) Frailty syndrome: A transitional state in a dynamic process. Gerontology 55: 539-549.

8. Cesari M, Landi F, Vellas B, Bernabei R, Marzetti E (2014) Sarcopenia and physical frailty: Two sides of the same coin. Front Aging Neurosci 6: 192.

9. Rochat S, Cumming RG, Blyth F, Creasey H, Handelsman D, et al. (2010) Frailty and use of health and community services by community-dwelling older men: The concord health and ageing in men project. Age Ageing 39: 228-233.

10. Xiaowei S, Mitnitski A, Rockwood K (2010) Prevalence and 10-year outcomes of frailty in older adults in relation to deficit accumulation. J Am Geriatr Soc 58: 681-687.

11. Sánchez-García S, Sánchez-Arenas R, García-Peña C, Rosas-Carrasco O, Ávila-Funes JA, et al. (2014) Frailty among community-dwelling elderly Mexican people: Prevalence and association with sociodemographic characteristics, health state and the use of health services. Geriatr Gerontol Int 14: 395-402.

12. Xue Q-L (2011) The frailty syndrome: Definition and natural history. Clin Geriatr Med 27: 1-15.

13. Heuberger RA(2011) The frailty syndrome: Acomprehensive review. J Nutr Gerontol Geriatr 30: 315-368.

14. Hoogendijk EO, van Hout HPJ, Heymans MW, van der Horst HE, Frijters DHM, et al. (2014) Explaining the association between educational level and frailty in older adults: Results from a 13-year longitudinal study in the Netherlands. Annals of Epidemiology 24: 538-544.

15. Castrejón-Pérez RC, Borges-Yáñez SA (2014) Frailty from an oral health point of view. J Frailty Aging 3: 180-186.

16. Rodríguez-Mañas L, Féart C, Mann G, Viña J, Chatterji $S$, et al. (2013) Searching for an operational definition of frailty: A delphi method based consensus statement. The frailty operative definition-consensus conference project. $J$ Gerontol A Biol Sci Med Sci 68: 62-67.

17. Mitnitski AB, Mogilner AJ, Rockwood K (2001) Accumulation of deficits as a proxy measure of aging. Scientific World Journal 1: 323-336.

18. Saum K-U, Dieffenbach AK, Müller H, Holleczek B, Hauer $\mathrm{K}$, et al. (2014) Frailty prevalence and 10-year survival in community-dwelling older adults: Results from the ESTHER cohort study. Eur J Epidemiol 29: 171-179.

19. Cacciatore F, Testa G, Galizia G, Della-Morte D, Mazzella F, et al. (2013) Clinical frailty and long-term mortality in elderly subjects with diabetes. Acta Diabetol 50: 251-260.

20. Topinková E (2008) Aging, disability and frailty. Ann Nutr Metab 52: 6-11. 
21. Collard RM, Boter H, Schoevers RA, Voshaar RCO (2012) Prevalence of frailty in community-dwelling older persons: A systematic review. J Am Geriatr Soc 60: 1487-1492.

22. Perkisas S, Vandewoude M (2014) Where frailty meets diabetes. Diabetes Metab Res Rev 32: 261-267.

23. Bouillon K, Kivimaki M, Hamer M, Sabia S, Fransson El, et al. (2013) Measures of frailty in population-based studies: An overview. BMC Geriatr 13: 64.

24. Buta BJ, Walston JD, Godino JG, Park M, Kalyani RR, et al. (2017) Frailty assessment instruments: Systematic characterization of the uses and contexts of highly-cited instruments. Ageing Res Rev 26: 53-61.

25. Ivan A, de Castro Cezar NO, Izbicki R, Lin SM, Paulo DLV, et al. (2017) Screening for frailty with the FRAIL scale: A comparison with the phenotype criteria. J Am Med Dir Assoc 18: 592-596.

26. van Kan GA, Rolland YM, Morley JE, Vellas B (2008) Frailty: Toward a clinical definition. J Am Med Dir Assoc 9: 71-72.

27. Abellan van Kan G, Rolland Y, Bergman H, Morley JE, Kritchevsky SB, et al. (2008) The I.A.N.A task force on frailty assessment of older people in clinical practice. J Nutr Health Aging 12: 29-37.

28. Rockwood K, Hogan DB, MacKnight C (2000) Conceptualization and measurement of frailty in elderly people. Drugs Aging 17: 295-302.
29. Batistoni SST, Neri AL, Cupertino APFB (2007) Validity of the center for epidemiological studies depression scale among Brazilian elderly. Rev Saude Publica 41: 598-605.

30. Park SW, Goodpaster BH, Strotmeyer ES, Rekeneire ND, Harris TB, et al. (2006) Decreased muscle strength and quality in older adults with type 2 diabetes: The health, aging, and body composition study. Diabetes 55: 18131818.

31. Seo Y-M, Hahm JR, Kim T-K, Choi W-H (2015) Factors affecting fatigue in patients with type II diabetes mellitus in Korea. Asian Nurs Res 9: 60-64.

32. Jung H-W, Kim S-W, Ahn S, Lim J-Y, Han J-W, et al. (2014) Prevalence and outcomes of frailty in Korean elderly population: Comparisons of a multidimensional frailty index with two phenotype models. PLoS One 9: e87958.

33. Woo J, Leung J, Morley JE (2012) Comparison of frailty indicators based on clinical phenotype and the multiple deficit approach in predicting mortality and physical limitation. J Am Geriatr Soc 60: 1478-1486.

34. Jung H-W, Jang I-Y, Lee YS, Lee CK, Cho E-I, et al. (2016) Prevalence of frailty and aging-related health conditions in older Koreans in Rural Communities: A cross-sectional analysis of the aging study of Pyeongchang Rural Area. J Korean Med Sci 31: 345-352. 